\title{
Improvement of Brain Tumor Feature based Segmentation using Decision based Alpha Trimmed Global Mean Filter
}

\author{
Pratibha Sharma \\ P.G Student, Department of ECE, \\ Regional Campus, Gurdaspur
}

\author{
Harjit Singh \\ Assistant Professor, Department of ECE, \\ Regional Campus, Gurdaspur
}

\begin{abstract}
Detection of the brain tumor is an important application of medical image processing. The literature survey in this paper has shown that the many of the existing methods has unobserved the deprived quality images like images with amount of noise or poor brightness. Moreover the much of the existing work on tumor detection has abandoned the use of object based segmentation. The overall goal of this research work is to propose an efficient brain tumor detection using the feature detection androundness metric. To enhance the tumor detection rate further we have integrated the proposed object based tumor detection with the Decision based alpha trimmed global mean. The proposed technique has the ability to produce effective results even in case of high density of the noise.
\end{abstract}

\section{Keywords}

Image Segmentation, Brain Tumor, MRI

\section{INTRODUCTION}

Brain MRI image segmentation is one of the most important applications of image segmentation technique, and is an important part of clinical diagnostic tools. Segmented image can help physicians to identify tumor tissues in brain, and monitor effectiveness of chemotherapy treatments. Image segmentation is to group pixels into regions for future process. In each partitioned region of an image, pixels have similar characteristics based on given criteria. Brain MRI image segmentation is one of the most important applications of image segmentation technique, and is an important part of clinical diagnostic tools. Physicians can use magnetic resonance images (MRI) to estimate volume of tumor tissues in brain before and after chemotherapy. The thickness of reconstruction of MRI slices is determined during scanning. If the size of tumor region in MRI can be measured, the volumetric estimation of tumor can be obtained by calculating the sum of the products of the slice thickness and the tumor region size of each MRI slice. Hence, segmenting the tumor region in MRI images becomes the key step in the procedure.

\section{METHODS OF IMAGE SEGMENTATION}

(a)Thresholding: The easiest technique of image segmentation is the thresholding technique. This technique is carrying a threshold value to convert a gray-scale image into a binary image. The way of this process is to choose the threshold value once multiple-levels are chosen. An image is assumed to be divided into two parts: foreground and background. The interesting objects in the image are a foreground and the rest is a background. Threshold $\mathrm{T}$ is first finalized by analyzing all the pixel intensity. Consider a pixel $\mathrm{f}(\mathrm{x}, \mathrm{y})$ classification is done as,

If $f(x, y)=\left\{\begin{array}{l}>_{T} ; \text { foreground } \\ <T ; \text { background }\end{array}\right.$

Problem associated with the thresholding technique is that it will ignore the spatial information of the pixel values and hence they are inefficient for images that blur at object boundaries.

(b) Compression-Based Method: Compression based methods propose that the best segmentation is the one that minimizes the general probable segmentations and the coding length of the data. The relationship between both concepts is that segmentation attempts to recover patterns in an image and any reliability in the image can be used to condense it. The system explains every segment by its texture and boundary shape. All of these mechanisms are represented by a probability distribution function and its coding length is figured as follows:

i) The boundary encoding influences the detail that regions in normal images tend to have a level contour. This preceding is expended by Huffman coding to instruct the difference chain code of the contours in an image. If the smooth an edge is the shorter coding length it achieves.

ii) Texture is prearranged by lossy-compression in a system parallel to description length (MDL) principle, but here the length of the data identified to the model is approximated by the number of samples times the entropy of the representation. The texture in every section is represented by a multivariate common allocation whose entropy has congested form appearance.

(c) Histogram-Based Method: Histogram-based methods are extremely well-organized as evaluate to additional segmentation techniques since they normally want only single exceed in the course of the pixels. In this method, a histogram is figured from all of the pixels in the image, and the peaks and valleys in the histogram are used to establish the clusters in the image. The histogram can be prepared in several modes when various frames are measured.. The histogram can moreover be valuable on a per pixel basis where the information outcome is used to verify the most frequent color for the pixel location. This method segments support on dynamic objects and a static location and it's ensuing in an unusual kind of segmentation helpful in video tracking. Individual weakness of the histogram-seeking 
technique is that it might not be easy to distinguish important peak and valley in the image.

(d) Edge Detection: Edge detection is a strong field on its own inside image processing. Region boundaries and edges are directly connected, because there is frequently a quick adjustment in strength at the area boundaries. Edge detection algorithms have consequently been used as the base of another segmentation method. The edges recognized by edge detection are frequently detached. To segment a thing from an image through, one desires congested region boundaries. The desired edges are the boundaries between such objects or spatial-taxons.

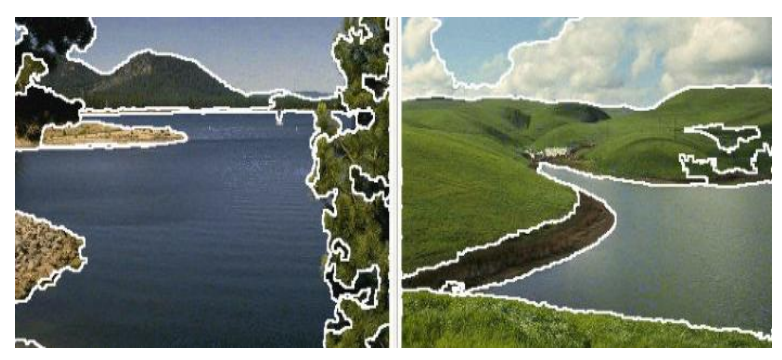

Fig 1: Edge Detection Image

(e) Region-Growing Method: Region-growing methods mostly rely on the statement that the adjacent pixels within individual region have related value.

One region-growing process was the seeded region growing process. This process gets a group of seeds as input alongside through the image. The seeds stain each of the objects to be segmented. The regions are iteratively developed by evaluating all unallocated adjacent pixels to the regions. The pixel with the minimum variation calculated this way is appropriate to the respective region. This process carried on till then each and every one pixel is payable to a region. Because seeded region growing needs seeds as extra input, the segmentation outcomes are reliant on the preference of seeds, and noise in the image can reason the seeds to be weakly placed different region-growing process was the unseeded region growing process. It is an adapted method that doesn't involve clear seeds. At every iteration, it deliberates the adjacent pixels exactly the same like as seeded region growing. It fluctuates from seeded region growing in that if the smallest amount of $\delta$ is less as compared to predefined threshold $\mathrm{T}$ then it is added to the particular region.

(f) Split and Merge method: This technique begins at the root of the tree that signified the complete image. If it is created non-uniform (not homogeneous), then it is divide into four son-squares (the splitting process), and so on so onwards. On the other hand, if these son-squares are identical, they are able to be combined as a number of associated components (the merging process). The joint in the tree is a segmented joint. This process carries on self repeating until no extra splits or merges are achievable. Mainly in this method first the splitting of four disjoint quadrants is prepared. As no other splitting is probable adjacent regions that are region with similar strength value is merged. It stops when no further splitting is achievable.

(g) Graph Partitioning Method: Graph partitioning process can efficiently be applied for image segmentation. In these schemes, the image is represented as a weighted, purposeless graph. Generally a pixel or a set of pixels are associated with joints and edge weights describe the resemblance and difference between neighboring pixels. The graph or image is then divided according to a condition planned to represent "good" clusters. It is mainly applied to a family of computational troubles in which the vertices of a graph have to be divided into two or extra large pieces as reduce the number of the edges that cross the cut

\section{LITERATURE SURVEY}

Prastawa, Marcel et al. [1] described some sort of design for auto neural unknown growth segmentation through MR images. The particular detectors connected with edema are performed concurrently by using unknown growth segmentation, because know-how from the level connected with edema is actually vital for examination, planning, plus treatment. Whilst a number of other unknown growth segmentation strategies rely on a depth augmentation generated with the gadolinium form a contrast adviser inside the T1-weighted image, the method offered in this article doesn't need form a contrast superior image channels. The only needed insight for your segmentation treatment is definitely the T2 MR image route, but it can make apply associated with a additional non-enhanced image programs for much better cells segmentation. The particular segmentation design is composed of a few stages. Initially, they find abnormal zones working with an authorized neural atlas because one for healthy and balanced brains. They then utilize the solid quotes connected with the spot plus dispersion from the regular neural cells depth groups to discover the depth attributes connected with the different cells types. Inside the 2nd phase, they determined in the T2 image intensities whether or not edema shows up combined with unknown growth inside the abnormal regions. Last but not least, they used geometric plus spatial limits to your found unknown growth plus edema regions. The particular segmentation treatment has been put on a few authentic datasets, which represents diverse unknown growth forms, destinations, styles, image intensities, plus enhancement. Zook, Justin M., plus Khan M. Iftekharuddin [2] prolonged their particular earlier perform by way of mathematically validating final results connected with FD study on a collection of 80 authentic MR plus CT images. Their own half-image approach needs that this unknown growth is situated in $50 \%$ from the neural while their particular wholeimage approach may not. Additionally, they took care of the necessity for some sort of personal reference (control) non tumor image to calculate a unknown growth FD, that was necessary within their earlier work. In addition, they when compared to the brain unknown growth detectors functionality of these algorithms with other fractal-based algorithms inside the materials plus mathematically examine their particular effects versus physically segmented unknown growth images. They will found that a unknown growth region gives a mathematically sizeable reduce FD compared to that of a non unknown growth location for many of the FD algorithms learned in this particular work. Therefore, their particular precise study shows that these types of FD algorithms might be exploited efficiently to discover the attainable position and venue connected with neural growths inside MR plus CT images. Murugesan, M., R. Sukanesh [3] provided an automated method for efficient detectors connected with neural growths inside EEG signs working with manufactured neurological cpa affiliate networks (ANNs). The particular ANN used in a offered method is give food to send back again propagation neurological network. Commonly, a EEG signs will likely comprise a selection of items through both equally theme plus devices items in the 
way in addition to vital the specifics of irregularities plus neural exercise (responses to a particular stimuli). Originally, versatile filtration is actually put on take away the items found in a EEG signal. Hereafter, common options found in a EEG indicate are removed working with spectral estimation. Precisely, spectral study is actually reached by employing Quickly Fourier Transform of which components a indicate options interred inside a vast range of noise. The particular clean up EEG facts consequently acquired is utilized because teaching insight to your give food to send back again propagation neurological network. The particular educated give food to send back again propagation neurological system if raised on by using a test EEG indicate, effectively detects the use of neural unknown growth inside the EEG signal. The particular trial and error effects prove the effectiveness of a offered method inside items treatment plus neural unknown growth detectors. Harati, Vida et al. [4] suggested an entirely computerized and also correct method for tumour area detectors and also segmentation inside mind magnet resonance (MR) images. A introduced solution is definitely an improved fuzzy connectedness (FC) algorithm formula with different range when the starting position is definitely chosen automatically. This specific algorithm formula is definitely separate with the tumour kind with regard to the pixels intensity. Cancer segmentation examination final results determined by similarity conditions (similarity index $(S I)$, overlap fraction $(O F)$, and other fraction (EF) usually are $92.89 \%, 91.75 \%$, and also $3.95 \%$, respectively) indicate a better operation with the proposed solution when compared to fliers and business cards, especially in MR illustrations or photos, inside tumour places along with lower contrast. Thus, the suggested strategy is wonderful for raising ale computerized calculate involving tumor measurement and also position inside mind tissues, that provides more accurate examination with the necessary surgical treatment, radiation treatment, and also radiotherapy procedures. Parisot, Debbie et al. [5] proposed your fresh solution for detectors, segmentation and also depiction of mind tumors. Its strategy exploits previous awareness by using your thin graph and or chart that represent the likely spatial jobs of tumor classes. Such information is definitely in addition to image-based classification procedures along with spatial level of smoothness limitations toward to become a reputable detection chart in just a unified graphical style formulation. In direction of maximum usage of previous awareness, a couple part connected graph and or chart is considered along with a single part equivalent for the lowgrade glioma kind (characterization) plus the 2nd part to help voxel-based decisions of tumor presence. Useful linear development each with regard to operation plus in terms of computational fill is considered to extract the lowest probable involving the target function. A result of the process identifies both tumor segmentation as well as characterization. Ensuring final results with significant information packages demonstrate the non plus ultra potentials of their total method. Subashini, M. Monica, and also Sarat Kumar Sahoo [6] talked about that the Brain tumor detection is an important program inside recent days. Professional medical troubles are intense if tumor is definitely discovered on the later on stage. Therefore a diagnosis is necessary on the earliest. MRI may be the present-day technological innovation which makes it possible for the detectors, a diagnosis and also evaluation. In the work, the wonderful pictures obtained by means of MRI usually are segmented and then provided to help one generally known as Heart beat coupled nerve organs network regarding uncovering the position of tumor inside the brain image. Your physician could possibly seek the help on this style in case the input MRI brain illustrations or photos tend to be inside amount plus the network would help your doctor to help not waste time additional analysis. The task also makes use of back propagation network regarding classification. The communities usually are much less complicated by nature and therefore the handling involving MRI brain illustrations or photos can be quite simple. A network categorizes the input illustrations or photos normally and tumor containing. The tumor could be civilized and also cancer and also it requires professional medical help additional classification. Maiti, Ishita, and also M. Chakraborty [7] developed a brand new strategy for brain tumor detection. For this specific purpose watershed strategy is required in combination with edge detection operation. It's a color based brain tumor detection algorithm formula working with color brain MRI illustrations or photos inside HSV color space. A RGB photo is definitely transformed into HSV color photo by which the style is definitely segregated inside three places shade, saturation, and also intensity. Right after form a contrast development watershed algorithm formula is definitely applied to the style for each and every region. Canny edge detector is definitely applied to the output image. Right after pairing several illustrations or photos final brain tumor segmented photo is definitely obtained. A algorithm formula has been placed onto twenty brain MRI images. Formulated algorithm formula offers granted offering results. Ghanavati, Sahar et al. [8] introduced your multi-modality framework regarding automatic tumor detectors, fusing various Permanent magnet Resonance Imaging modalities such as T1weighted, T2-weighted, and also T1 along with gadolinium form a contrast agent. A high intensity, contour deformation, balance, and also consistency attributes ended up taken from every image. A AdaBoost classifier was utilized to help find the nearly all discriminative attributes and to segment the tumor region. Multi-modal MR illustrations or photos along with simulated tumor happen to be utilised while the land simple fact regarding training and also consent involving the detectors method. Preliminary final results with simulated and also patient MRI indicate $100 \%$ successful tumor detectors along with common accuracy and reliability involving $90.11 \%$. Vijay, J., and J. Subhashini [9] discussed segmentation of images holds an important position in the area of image processing. It becomes more important while typically dealing with medical images where presurgery and post surgery decisions are required for the purpose of initiating and speeding up the recovery process. Computer aided detection of abnormal growth of tissues is primarily motivated by the necessity of achieving maximum possible accuracy. Manual segmentation of these abnormal tissues cannot be compared with modern day's high speed computing machines which enable us to visually observe the volume and location of unwanted tissues. A well known segmentation problem within MRI is the task of labeling the tissue type which include White Matter (WM), Grey Matter (GM), Cerebrospinal Fluid (CSF) and sometimes pathological tissues like tumor etc. This paper describes an efficient method for automatic brain tumor segmentation for the extraction of tumor tissues from MR images. In this method segmentation is carried out using K-means clustering algorithm for better performance. This enhances the tumor boundaries more and is very fast when compared to many other clustering algorithms. The proposed technique produce appreciative results. Ulku, Eyup Emre, and Ali Yilmaz Camurcu [10] described the CAD system that is based on histogram equalization and morphological image processing techniques. The processes are carried out through 125 MR images which are taken from 11 people that are 8 
people with tumors, 3 people without tumors. In classification stage which is the last stage of the computeraided detection systems, 6 classification algorithms are tested in the rapid miner program, and these algorithms are compared with each other to show CAD system accuracy. Halder, Amitava [11] proposed an efficient brain tumor detection method, which can detect tumor and locate it in the brain MRI images. This method extracts the tumor by using K-means algorithm followed by Object labeling algorithm. Also, some preprocessing steps (median filtering and morphological operation) are used for tumor detection purpose. It is observed that the experimental results of the proposed method gives better result in comparison to other techniques. Aswathy, S. U. et al. [12] have discussed that the Brain tumor detection plus segmentation is one of the most challenging and very time consuming task in medical processing. MRI has a medical technique, mainly utilized by the radiologist for visualization of internal structure of the human body without surgery. Accurate segmentation of MRI image is important for the diagnosis of brain tumor by computer aided clinical tool. After appropriate segmentation of brain MR images, tumor is classified to malignant and benign, which is a difficult task due to complexity and variation in tumor tissue characteristics its shape, size, gray level intensities and location. Taking in to account the aforesaid challenges, this research is focused highlighting the strength and limitations of earlier proposed classification techniques discussed in the contemporary literature. Besides summarizing the literature, the paper also provides a critical evaluation of the surveyed literature which reveals new facets of research. Preetha, R., and G. R. Suresh [13] discussed that the Image Segmentation is important and challenging to visualize the tissue of human for analyzing the MR images. In brain MR images, the boundary of tumor tissue is highly irregular. Deformable models and Region based methods are extensively used for medical image segmentation, to locate the boundary of the tumor. Problems connected with nonlinear distribution of real data, User interaction and poor convergence to the boundary region limited their usefulness. Clustering of brain tumor images, using Fuzzy $\mathrm{C}$ means is robust and effective for tumor localization. Even although proposed method has high computational complexity, it shows superior results in segmentation efficiency and convergence rate. The Fuzzy $\mathrm{C}$ means clustering with the extension of Feature extraction and classification is extremely promising in the field of brain tumor detection Njeh, et al. [14] looked into an effective distribution-matching, data-driven requirements to have 3D multimodal MRI thought function glioma unfamiliar growth and also edema segmentation in a range of modalities. Purchased right after modern-day boundrelaxation outcomes, the specific optima about the fee performs provide the specific boost about the unfamiliar growth district and also edema district with just pertaining to real-time. Determined by world as an alternative to pixel wise details, the specific prepared requirements isn't going to entail a fantastic alternative playing suggestions from the massive, manually-segmented instructing placed, since can be the case about the first sort methods. Consequently, the specific ensuing email address information and facts are outside of choosing getting some instructing set. Quantitative recommendations from the publicly available instructing and also screening data placed through the MICCAI multimodal thought function unfamiliar growth segmentation obstacle (BraTS 2012) revealed that their unique requirements assure the incredibly reasonably competitive overall performance to have total edema and also unfamiliar growth segmentation, including seeking pre-existing rivalling methods, together with the fascinating computing object rendering time period. Dhage, Padmakant et al. [15] explained alcohol watershed segmentation to separate the specific unnatural structure via the ordinary all-around structure to acquire a true recognition about employed and also noninvolved area that assist the surgeon to know away from each other the specific employed area precisely. Right after carrying out this unfamiliar growth is going to be removed from the specific MR picture and special spot and also style tend to be determined as well as other factors just like exterior, eccentricity, entropy and also centroid are actually calculated. Nabizadeh, Nooshin, plus Miroslav Kubat [16] introduced a completely computerized method, which usually may identify slices that come with unknown growth plus, so that you can delineate the actual unknown growth area. The actual experimental final results upon single comparison system show the actual effectiveness with their planned procedure with productively segmenting mental faculties unknown growth tissue with high exactness plus lower computational complexity.. AbdelMaksoud et al. [17] introduced a simple yet effective photo segmentation strategy making use of $\mathrm{K}$-means clustering procedure included together with Fluffy C-means algorithm. It truly is accompanied by thresholding plus place set segmentation steps to supply a precise mental faculties unknown growth detection. The actual planned procedure could get benefits associated with the actual K-means clustering to get photo segmentation inside areas of minimum computation time. In addition, it could get advantages of the Fluffy C-means inside areas of accuracy. The actual overall performance on the planned photo segmentation strategy appeared to be assessed by means of comparing it by incorporating cutting-edge segmentation algorithms in the instance of exactness, running time, plus performance. The accuracy appeared to be assessed by means of comparing the results together with the earth real truth of the refined image. The actual experimental final results describe the potency of their particular planned strategy to deal with a better volume of segmentation difficulties by means of improving the segmentation top quality plus exactness with minimum rendering time.

\section{PROPOSED METHODOLOGY}

This section contains the various steps to achieve the objectives of this research work: Figure 2 has shown various steps required to get the final image using the proposed algorithm. Subsequent are the various steps with brief detail:-

Step 1: Input Image: - First of image will be taken as an input.

Step 2: Apply decision based alpha trimmed filter:- In this step, filter will be applied to the input image to remove noise. The filter to be applied is named as decision based alpha trimmed filter.

Step 3: Apply image segmentation: - After filtration of input image, image is segmented.

Step 4: Apply feature based tumor detection:- After the image is segmented, feature based tumor detection is applied to the segmented image.

Step 5: In this step, tumor is detected from the Step 4 image.

Step 6: Evaluate performance parameters:- After detecting the tumor, various performance parameters are evaluated to find the proficiency of the proposed algorithm. 


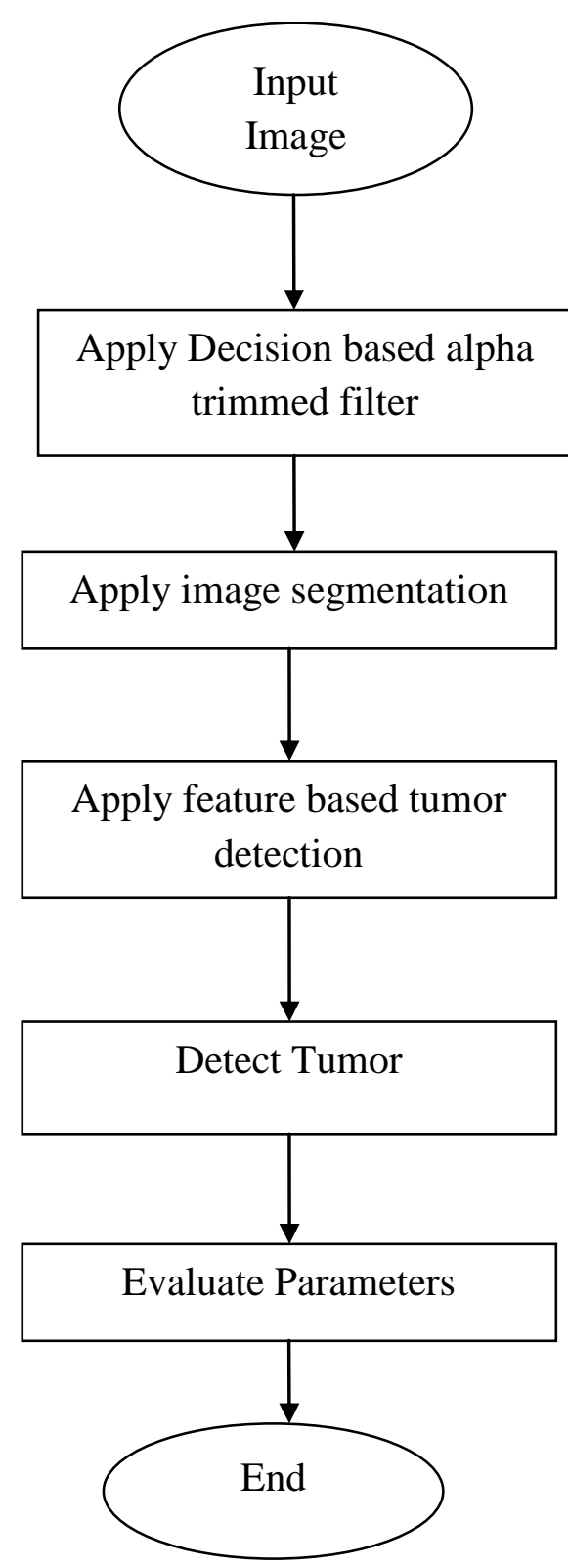

Fig 2: Flow chart of proposed algorithm

\section{RESULTS AND DISCUSSIONS EXPERIMENTAL SETUP}

In this section, we explain the results of the proposed methodology and its comparison with the existing techniques.

Figure 3 is the input image and its outputs are as shown below.

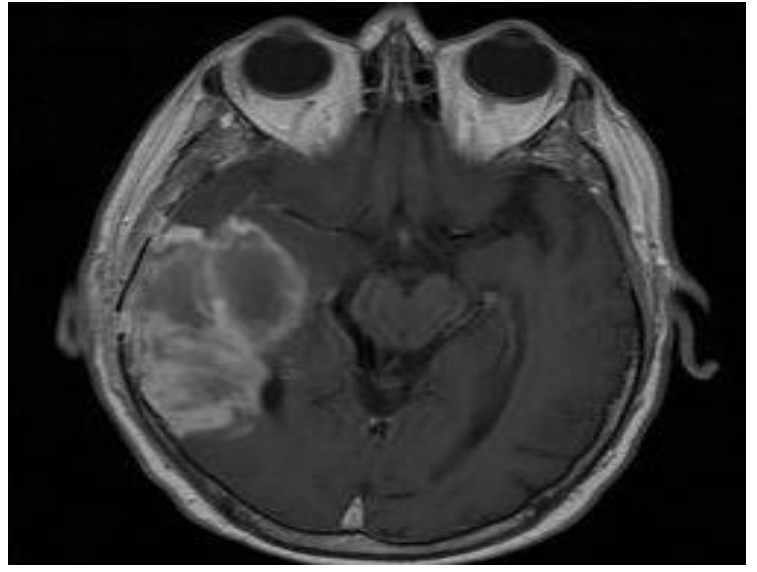

Fig 3: Input image

Figure 4 is the filtered image after applying Decision Based Alpha Trimmed Global Mean Filter

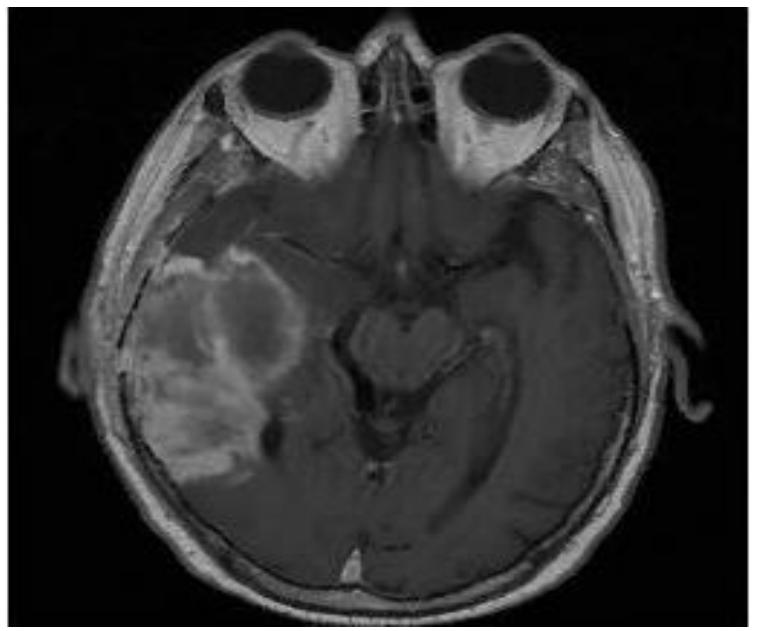

Fig 4: Filtered image

Figure 5 is the Binary Image Using OTSU Segmentation

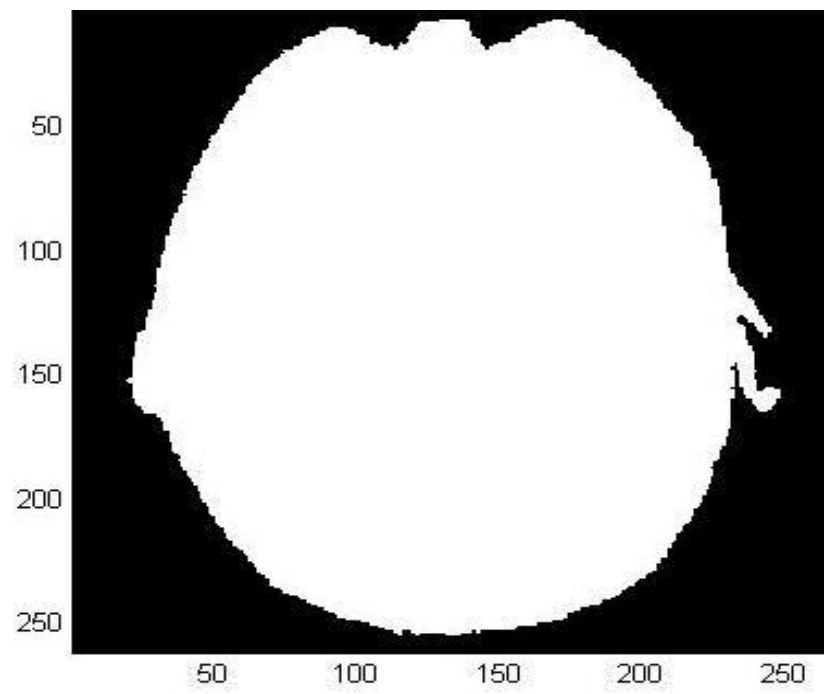

Fig 5: Binary Image Using OTSU Segmentation 
Figure 6 is the output of figure 5 after applying the Modified Hough Metric to Detect Segmented Object

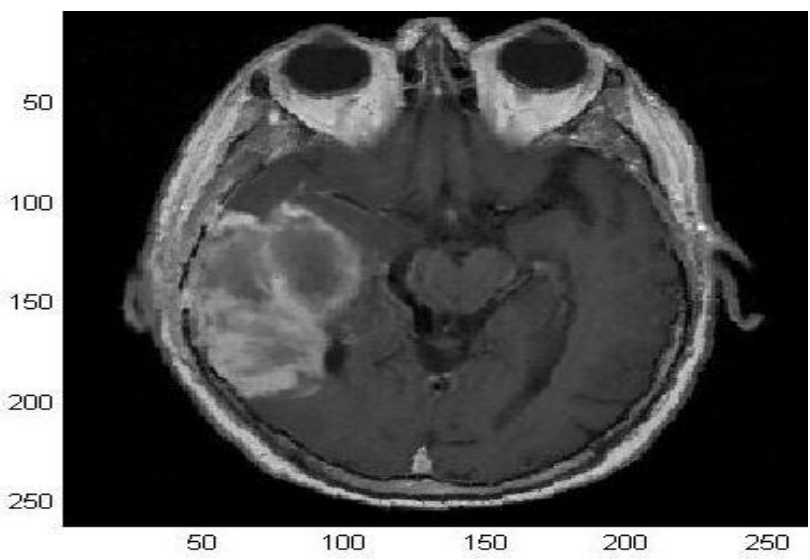

Fig 6: After applying the Modified Hough Metric to Detect Segmented Object

Figure 7 is the image after evaluating Segment with Highest Score

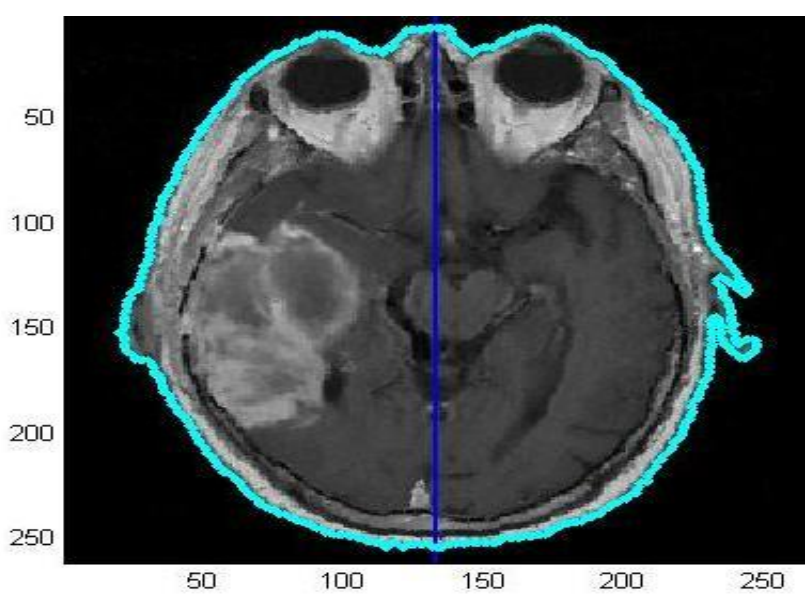

Fig 7: Image after evaluating Segment with Highest Score

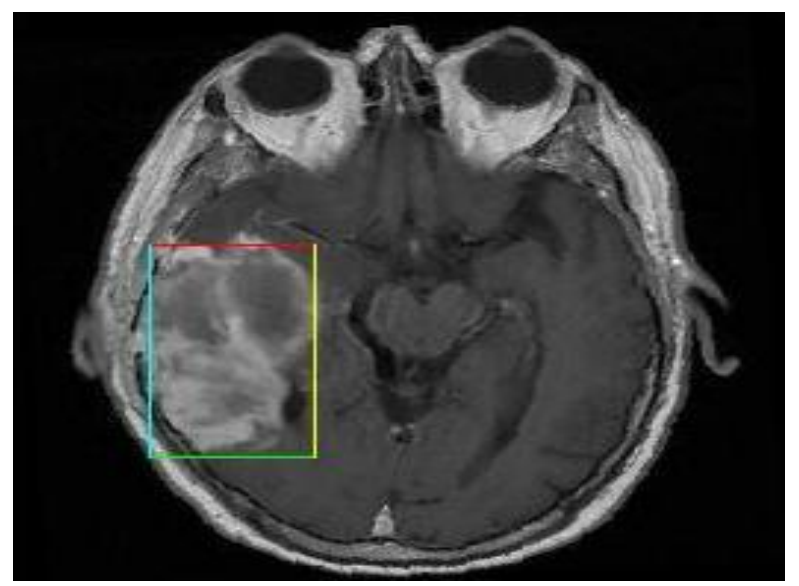

Fig 8: Image after tagging it As Tumor Area

\section{PERFORMANCE EVALUTION}

ACCURACY RATE: - Accuracy is the mean of sensitivity and specificity

$$
A=\frac{\sum \mathrm{T}_{\mathrm{p}}+\sum \mathrm{T}_{\mathrm{n}}}{\sum \mathrm{T}_{\mathrm{p}}+\sum \mathrm{T}_{\mathrm{n}}+\sum \mathrm{F}_{\mathrm{p}}+\sum \mathrm{F}_{\mathrm{n}}}
$$

$T_{p}$ is total positives, $T_{n}$ are total negatives, $F_{p}$ is falsel positives, $F_{p}$ are false negatives

Table 1 is showing the comparative analysis of the Accuracy. As Accuracy need to be maximized; so the main goal is to increase the F-measure as much as possible.

Table 1: Accuracy Evaluation

\begin{tabular}{|c|c|c|}
\hline $\begin{array}{c}\text { NOISE } \\
\text { DENSITY }\end{array}$ & PROPOSED & EXISTING \\
\hline 0.01 & 0.9946 & 0.1895 \\
\hline 0.02 & 0.9897 & 0.1754 \\
\hline 0.03 & 0.9852 & 0.1569 \\
\hline 0.04 & 0.9802 & 0.1466 \\
\hline 0.05 & 0.9752 & 0.2095 \\
\hline 0.06 & 0.9692 & 0.1207 \\
\hline 0.07 & 0.9672 & 0.1042 \\
\hline 0.08 & 0.9603 & 0.0962 \\
\hline
\end{tabular}

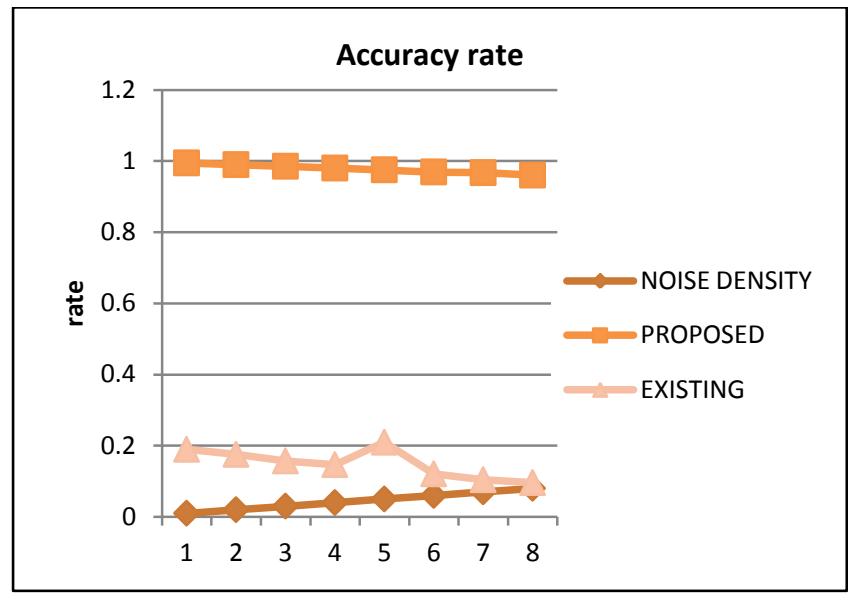

Fig 9: Accuracy Evaluation

Figure 9 has shown the quantized analysis of the accuracy of different. It is very clear from the plot that there is increase in f-measure value of images with the use of method over existing methods. This increase represents improvement in the objective quality of the image.

PSNR: The PSNR block computes the peak signal-to-noise ratio, between two images.

$$
P S N R=10 \log _{10}\left(\frac{255^{2}}{M S E}\right)
$$

Table 2 is showing the comparative analysis of the Peak Signal to Noise Ratio (PSNR). As PSNR need to be maximized; so the main goal is to increase the PSNR as much as possible. 
Table 2: PSNR Evaluation

\begin{tabular}{|c|c|c|}
\hline $\begin{array}{c}\text { NOISE } \\
\text { DENSITY }\end{array}$ & PROPOSED & EXISTING \\
\hline 0.01 & 45.3595 & 1.8251 \\
\hline 0.02 & 39.7435 & 1.6748 \\
\hline 0.03 & 36.5942 & 1.4820 \\
\hline 0.04 & 34.0775 & 1.3769 \\
\hline 0.05 & 32.1018 & 2.0415 \\
\hline 0.06 & 30.226 & 1.1175 \\
\hline 0.07 & 29.4025 & 0.9558 \\
\hline 0.08 & 28.0235 & 0.8785 \\
\hline & & \\
\hline
\end{tabular}

Figure 10 has shown the quantized analysis of the peak signal to noise ratio of different. It is very clear from the plot that there is increase in PSNR value of images with the use of method over existing methods.

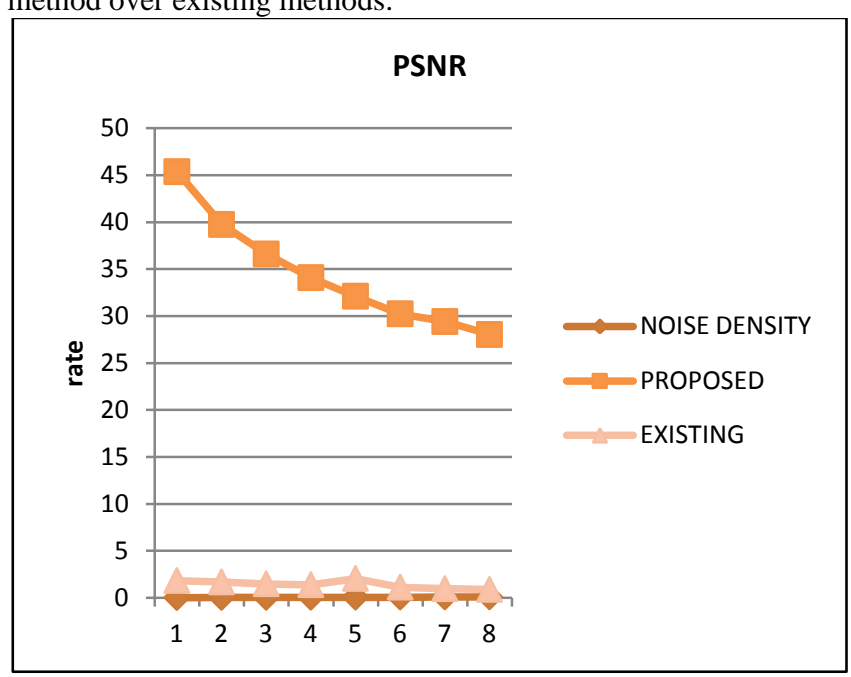

Figure 10: PSNR Evaluation

BIT ERROR RATE: The bit error rate or bit error ratio (BER) is the number of bit errors divided by the total number of transferred bits during a studied time interval.

$$
(B E R)=\frac{\text { Numbers of Errors }}{\text { Total number of bits sent }}
$$

Table 3 has clearly shown that the BER is minimum in the case of the algorithm; therefore algorithm is providing better results than the available methods.

Figure 11 has shown the quantized analysis of the bit error rate of different.

It is very clear from the plot that there is decrease in BER value of images with the use of method over existing methods.
Table 3: BER Evaluation

\begin{tabular}{|c|c|c|}
\hline Noise density & Proposed & Existing \\
\hline 0.01 & 0.5297 & 46.6404 \\
\hline 0.02 & 1.0969 & 46.6943 \\
\hline 0.03 & 1.5149 & 46.7621 \\
\hline 0.04 & 1.9835 & 46.7986 \\
\hline 0.05 & 2.4177 & 46.5615 \\
\hline 0.06 & 3.0431 & 46.8869 \\
\hline 0.07 & 3.2816 & 46.9408 \\
\hline 0.08 & 3.8216 & 46.9663 \\
\hline & & \\
\hline & & \\
\hline
\end{tabular}

BER

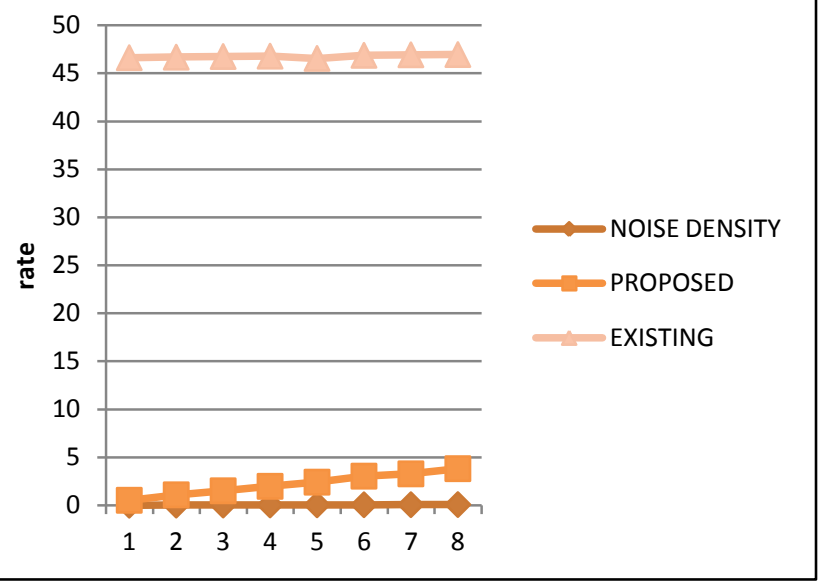

Figure 11: BER Evaluation

F-MEASURE: The F-Measure computes average of the information retrieval precision and recall metrics.

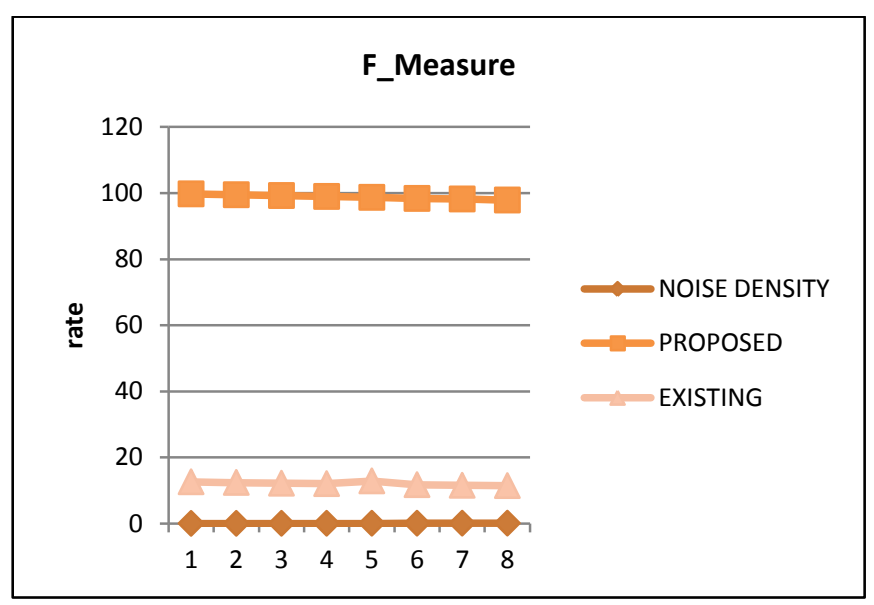

Figure 12: F-measure Evaluation 
Table 3: F-measure Evaluation

\begin{tabular}{|c|c|c|}
\hline Noise density & Proposed & Existing \\
\hline 0.01 & 99.7132 & 12.5922 \\
\hline 0.02 & 99.4519 & 12.4029 \\
\hline 0.03 & 99.2106 & 12.1638 \\
\hline 0.04 & 98.9428 & 12.0356 \\
\hline 0.05 & 98.6695 & 12.8689 \\
\hline 0.06 & 98.3434 & 11.7225 \\
\hline 0.07 & 98.1766 & 11.5312 \\
\hline 0.08 & 97.8567 & 11.4407 \\
\hline
\end{tabular}

Table 4 is showing the comparative analysis of the F-measure As F-measure need to be maximized; so the main goal is to increase the F-measure as much as possible.

\section{CONCLUSION AND FUTURE SCOPE}

In this paper, a new feature detection based tumor detection algorithm using roundness metric has been proposed. Moreover the proposed feature based tumor detection has been integrated with the Decision based alpha trimmed global mean filter to remove noise. The comparison in the paper shows the efficiency of the proposed technique over the existing technique on the basis of various performance metrics.

\section{REFERENCES}

[1] Prastawa, Marcel, Elizabeth Bullitt, Sean Ho, and Guido Gerig. "A brain tumor segmentation framework based on outlier detection." Medical image analysis 8, no. 3 (2004): 275-283.

[2] Zook, Justin M., and Khan M. Iftekharuddin. "Statistical analysis of fractal-based brain tumor detection algorithms." Magnetic Resonance Imaging 23, no. 5 (2005): 671-678.

[3] Murugesan, M., and R. Sukanesh. "Automated detection of brain tumor in EEG signals using artificial neural networks." In Advances in Computing, Control, \& Telecommunication Technologies, 2009. ACT'09. International Conference on, pp. 284-288. IEEE, 2009.

[4] Harati, Vida, Rasoul Khayati, and Abdolreza Farzan. "Fully automated tumor segmentation based on improved fuzzy connectedness algorithm in brain MR images." Computers in biology and medicine 41, no. 7 (2011): 483-492.

[5] Parisot, Sarah, Hugues Duffau, Stéphane Chemouny, and Nikos Paragios. "Graph-based detection, segmentation \& characterization of brain tumors." InComputer Vision and Pattern Recognition (CVPR), 2012 IEEE Conference on, pp. 988-995. IEEE, 2012.
[6] Subashini, M. Monica, and Sarat Kumar Sahoo. "Brain Tumour Detection Using Pulse Coupled Neural Network (PCNN) and Back Propagation Network." (2012): 10-15.

[7] Maiti, Ishita, and M. Chakraborty. "A new method for brain tumor segmentation based on watershed and edge detection algorithms in HSV colour model." InNational Conference on Computing and Communication Systems, pp. 177-188. 2012.

[8] Ghanavati, Sahar, Junning Li, Ting Liu, Paul S. Babyn, Wendy Doda, and George Lampropoulos. "Automatic brain tumor detection in magnetic resonance images." In Biomedical Imaging (ISBI), 2012 9th IEEE International Symposium on, pp. 574-577. IEEE, 2012.

[9] Vijay, J., and J. Subhashini. "An efficient brain tumor detection methodology using K-means clustering algoriftnn." In Communications and Signal Processing (ICCSP), 2013 International Conference on, pp. 653657. IEEE, 2013.

[10] Ulku, Eyup Emre, and Ali Yilmaz Camurcu. "Computer aided brain tumor detection with histogram equalization and morphological image processing techniques." In Electronics, Computer and Computation (ICECCO), 2013 International Conference on, pp. 48-51. IEEE, 2013.

[11] Halder, Amitava, Chandan Giri, and Amiya Halder. "Brain tumor detection using segmentation based Object labeling algorithm." In Electronics, Communication and Instrumentation (ICECI), 2014 International Conference on, pp. 1-4. IEEE, 2014.

[12] Aswathy, S. U., Deva Dhas, G. Glan, and S. S. Kumar. "A survey on detection of brain tumor from MRI brain images." In Control, Instrumentation, Communication and Computational Technologies (ICCICCT), 2014 International Conference on, pp. 871-877. IEEE, 2014.

[13] Preetha, R., and G. R. Suresh. "Performance Analysis of Fuzzy C Means Algorithm in Automated Detection of Brain Tumor." In Computing and Communication Technologies (WCCCT), 2014 World Congress on, pp. 30-33. IEEE, 2014

[14] Njeh, Ines, Lamia Sallemi, Ismail Ben Ayed, Khalil Chtourou, Stephane Lehericy, Damien Galanaud, and Ahmed Ben Hamida. "3D multimodal MRI brain glioma tumor and edema segmentation: A graph cut distribution matching approach." Computerized Medical Imaging and Graphics (2014)

[15] Dhage, Padmakant, M. R. Phegade, and S. K. Shah. "Watershed segmentation brain tumor detection." In Pervasive Computing (ICPC), 2015 International Conference on, pp. 1-5. IEEE, 2015.

[16] Nabizadeh, Nooshin, and Miroslav Kubat. "Brain tumors detection and segmentation in MR images: Gabor wavelet vs. statistical features." Computers \& Electrical Engineering (2015).

[17] Abdel-Maksoud, Eman, Mohammed Elmogy, and Rashid Al-Awadi. "Brain tumor segmentation based on a hybrid clustering technique." Egyptian Informatics Journal 16, no. 1 (2015): 71-81. 